Historic, archived document

Do not assume content reflects current scientific knowledge, policies, or practices. 

LIBEARY

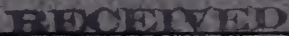

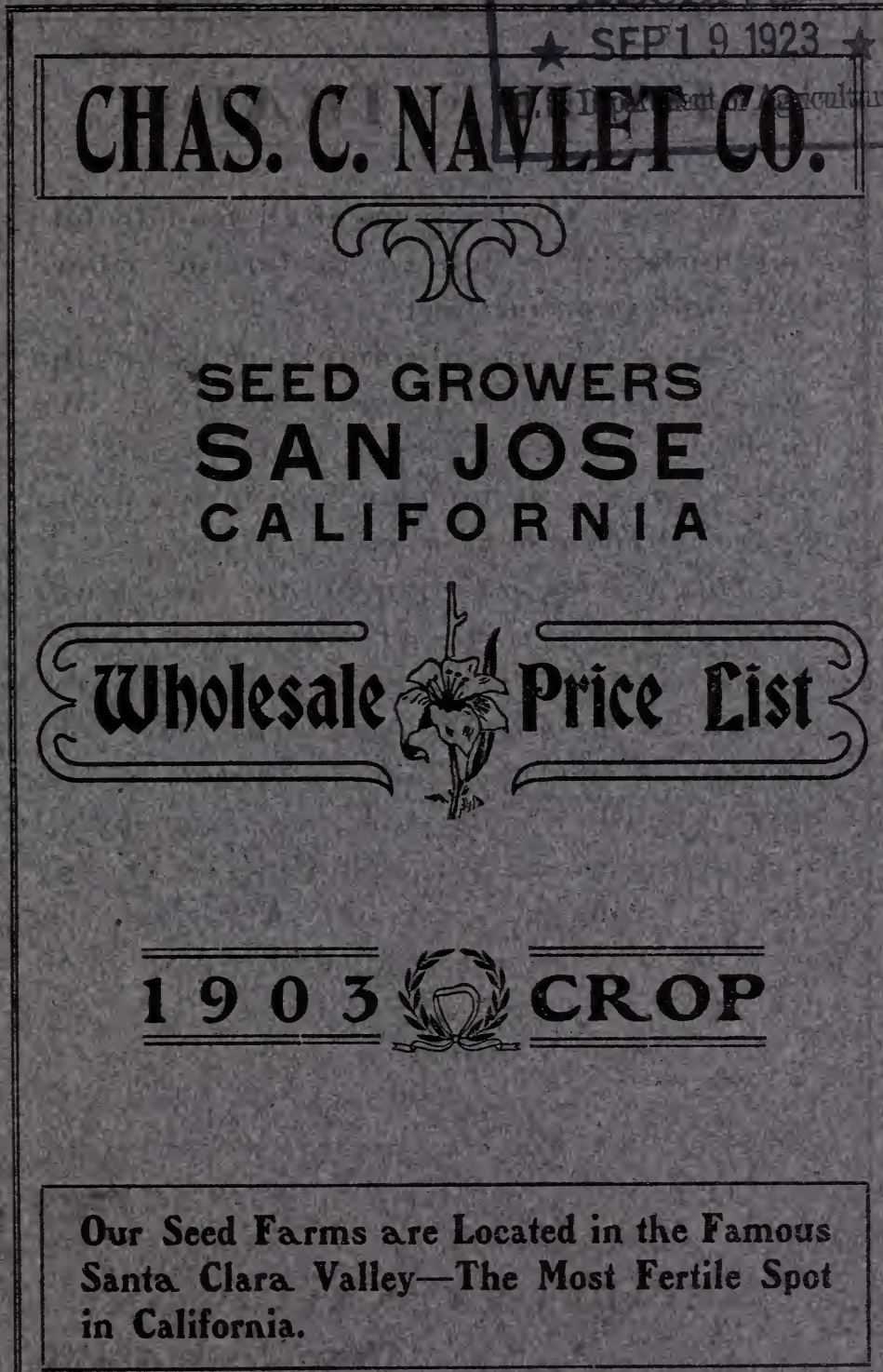




\section{TO THE TRADE}

We seek the business of all seed dealers and florists who believe in buying where their dollars go farthest.

Our seeds are absolutely reliable-the very best that it is possible to grow. We know this to be sa because all the seeds we sell are grown on our own seed farms under perfect climatic conditions.

Our prices are from 50 to $75 \%$ lower than those of any other house in the United States selling seeds of the same reliability. One reason for this is because we save our customers the middleman's profit-a big item. Another is because we chop every avoidable expense in the planting, harvesting and marketing of our seeds. And a third is because we are satisfied with very small profits.

Any seedsman who is not too busy to save money uan quickly convince himself of the proof of these statements by comparing the prices we quote on the following pages with those asked by other wholesale houses.

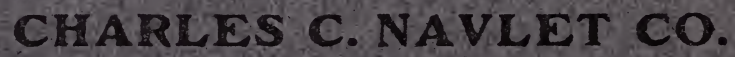

San Jose, Calif. 


\section{Charles C. Navlet Co.}

\section{$\frac{\text { SA N JOSE }}{\text { CALIEORNIA }}$}

GROWERS AND WHOLESALERS

* OF *

THE BEST FOOWER SEEDS IN THE WORLD

OLR IVORKING CAPITAL OF FIFTY THOUSAND DOLLARS TOGETHER WITH OLR UNSLRPASSED FACILITIES FOR MAKING PROMPT SHIPIENTS TO ALL PARTS OF THE COUNTRI AND THE THOROLGH KNOILEDGE OF THE BLSINESS THAT IVE HAYE GAINED BI TWENTY-FIVE IEARS OF CAREFUL, STLDY AND PRACTICAL LABOR AMONG THE PLANTS IN THE FIELD, PLACES US IN A.POSITION TO SATISFI THE DENANDS OF THE LARGEST BLYERS.

California Grown Seeds Mature Earliest and Bloom Most Luxuriantly. 

C H A R L E S
C. NAVLET
COMPANY
S A N J O S E,

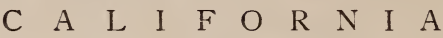

\section{NAVLET'S SWEET PEAS}

THE BEST IN THE WORLD.

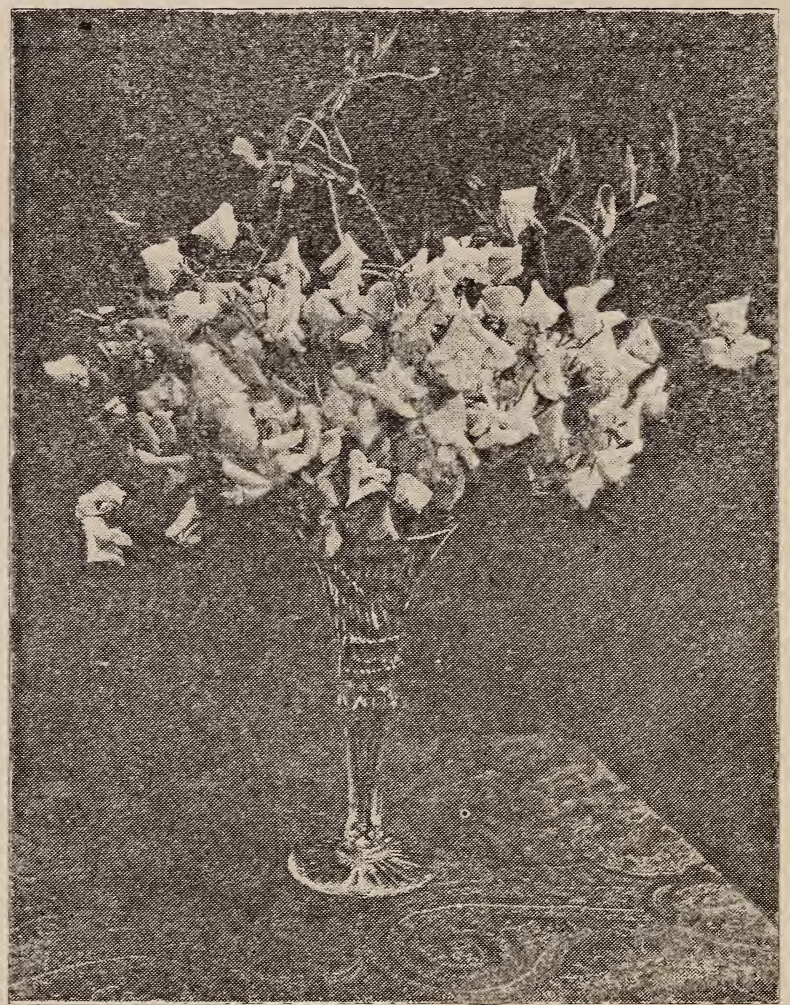

These Prices Hold Good Until Our Stock is EX, hausted. On Account of the Short Crops This Year We Would Advise You To Send Us Your Orders Early. 


\section{CHARLES C. NA VLET COMPA N Y,

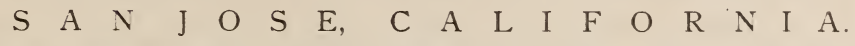

\section{NAVLET'S SWEET PEAS} THE BEST IN THE WORLD

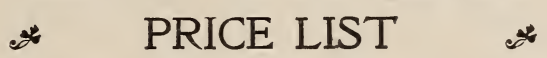

THIS LIST has been carefully selected and covers the entire range of colors and shades, all of them being of the large flowering type and of good form and substance:

Those varieties especially suited for cut flowers and floral work are indicated by a "*" opposite them.

PRICES POST PAID

LB. $1 / 4 \mathrm{LB} . \mathrm{OZ}$.

Admiration, a rosy lavender, ........... 3 30 Iос $5 \mathrm{C}$

America, blood red, striped ........... 3 Oc IOC $5 \mathrm{C}$

Apple Blossom, bright rose pink,........ 30 IOC $5 \mathrm{C}$

Aurora, white, striped with orange pink,.... 30c IOc $5 \mathrm{C}$

Blanche Burpee, purest white........... $30 \mathrm{C}$ Ioc $5 \mathrm{c}$

Blanche Ferry Extra Early, for forcing......* $30 \mathrm{C}$ roc $5 \mathrm{C}$

Burpee's New Countess, best lavender.......* 30c Ioc $5 \mathrm{C}$

Burpee's Sunproof Salopian, best scarlet.....* $30 \mathrm{O}$ Iоc $5 \mathrm{C}$

Captain of the Blues, light purple wings..... 30 IOC $5 \mathrm{C}$

Coquette, yellow and lavender............ 30 с Iо $5 \mathrm{C}$

Countess of Radnor, fine lavender........... 30

Crown Jewel, violet, rose and primrose..... 30 Ioc $5 \mathrm{C}$

Cupid Alice Eckford................ 30 IOC 5 C

Cupid Beauty.................. 30 IOC $5 \mathrm{C}$

Cupid Boreatton.................. 30 IOC

Cupid Countess of Radnor............. 30 IOC $5 \mathrm{C}$

Cupid Firefly.................. 30с Ioc $5 \mathrm{c}$

Cupid Primrose.................. 3 oc Ioc $5 \mathrm{c}$

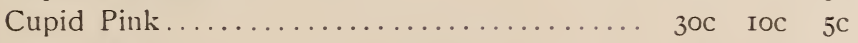

Cupid White.................... $30 \mathrm{C}$ IOC $5 \mathrm{C}$

Cupid Mixed ................... 3 oc Ioc $5 \mathrm{C}$

Duke of Clarence, claret............. 30 IOC $5 \mathrm{C}$

Dorothy Tennant, mauve............ 3 oc Ioc $5 \mathrm{c}$

Emily Eckford, reddish, mauve and blue.....* $30 \mathrm{C}$ Ioc $5 \mathrm{C}$

California Grown Seeds Mature Earliest and Bloom Most Luxuriantly. 


\section{CHA R L E C. NA V L E T COMPA N Y

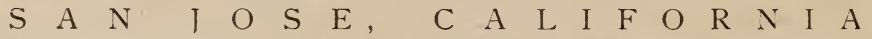

\section{SWEET PEA LIST CONTINUED}

PRICES POST PAID

Hon. F. Bouverie, flesh pink ......... 300 IOC 5

Katherine Tracy, soft pink............ 3 OO IOC $5 \mathrm{C}$

Lady Mary Currie, best orange pmk........ 3 OC Ioc $5 \mathrm{C}$

Lady Nina Balfour, delicate mauve........ 30с Ioc $\overline{5} \mathrm{C}$

Lady Grisel Hamilton, pale lavender........ $30 \mathrm{C}$ Iос $5 \mathrm{C}$

Lottie Hutchins, cream and pink......... 30 Ioc $5 \mathrm{c}$

Lottie Eckford, white shaded lavender...... 30 30 Ioc $5 \mathrm{C}$

Lovely, soft shell pink...............* $30 \mathrm{C}$ Iос $5 \mathrm{c}$

Maid of Honor, white, edge blue........* 30 1 oc $5 \mathrm{C}$

Mars, bright scarlet............... 30 Ioc $5 \mathrm{C}$

Modesty, very delicate pink........... 30c Ioc $5 \mathrm{C}$

Mrs. Dugdale, deep rose, fine........... $30 \mathrm{C}$ Ioc $5 \mathrm{C}$

Mrs. Eckford, primrose yellow........... * 30i Ioc 5c

Mrs.Joseph Chamberlain, rose, striped white.. 300 Ioc $5 \mathrm{C}$

Navy Blue, deep violet blue............. $30 \mathrm{c}$ 10с $5 \mathrm{c}$

Othello, deep maroon............... $30 \mathrm{C}$ IOC $5 \mathrm{C}$

Prima Domna, fine light pink ..........* 3 Oc Ioc $_{-5}^{-15}$

Prince Edward of York.............. 30 IOC $5 \mathrm{C}$

Prince of Wales, scarlet and rose.........* 30c IOC $5 \mathrm{C}$

Queen Victoria, primrose with purple......* $30 \mathrm{O}$ IOC $5 \mathrm{C}$

Famona, white splashed pink.......... 30 Iоc $5 \mathrm{C}$

Royal Rose, deep rose pink ............ $30 \mathrm{C}$ Iос $5 \mathrm{C}$

Sadie Burpee, pure white ............. $30 \mathrm{c}$ Iос $5 \mathrm{c}$

Salopian, fine scarlet.............. 30 Ioc $5 \mathrm{c}$

Sensation, white, suffused pink......... 30c Ioc $5 \mathrm{C}$

Shahzada, deep purple .............. 30с Ioc 5 c

Stanley, dark mauve.............. 30

Stella Morse, primrose flushed pink........ $30 \mathrm{C}$ Iос $5 \mathrm{C}$

Triumph, orange pink.............. 30 IOC $5 \mathrm{C}$

Venus, salmon buff pink............. 3 Oc Iос $5 \mathrm{C}$

Mixed Choice Double............... 30 . Ioc $5 \mathrm{C}$

Mixed, Eckford Hybrid, choice mixed...... 25C Ioc $5 \mathrm{C}$

Mixed, California Giant, the very finest...... $30 \mathrm{C}$ Ioc $5 \mathrm{C}$

California Grown Seeds Mature Earliest and Bloom Most Luxuriantly. 


\section{CHARLES C. NAVLET COMPANY $\begin{array}{lllllllllllllllll}S & A & N & J & O & S & E, & C & A & L & I & F & O & R & N & \text { I } & A\end{array}$}

\section{NAVLET'S ASTERS}

\section{THE BEST IN THE WORLD \\ PRICE LIST}

Giant Branching Comet, Carmine,...

Giant Branching Comet, Rose-pink.

Giant Branching Comet, White ......

Giant Branching Comet, White, striped

Giant Branching Comet, IIixed........

Semples, Branching Crimson........

Semples, Branching Carnine.........

Semples, Branching Lavender.......

Semples, Branching Pink............

Semples, Branching Purple..........

Semples, Branching Rose..........

Semples, Branching White..........

Semples, Branching Mixed..........

Dwarf Comet, Mixed...............

Giant Comet, Mixed................

Giant Comet, White...............

Truffaut's Pæony Flowered Perfection,

Nixed ...................

Improved Victoria, Mixed..........

Queen of the Market, Mixed.........
PRICES POST PAID

th $\quad \mathrm{I} / \mathrm{Hb} \quad \mathrm{OZ}$. $\$ 7.00 \quad \$ 2.50 \quad 70 \mathrm{C}$

$7.00 \quad 2.50 \quad 70 \mathrm{OC}$

$7.00 \quad 2.50 \quad 70 \mathrm{C}$

$7.00 \quad 2.50^{\circ} \quad 70 \mathrm{C}$

$6.00 \quad 2.00 \quad 60 \mathrm{C}$

$4.50 \quad$ I.5O $40 \mathrm{C}$

$4.50 \quad I .50 \quad 40 C$

$4.50 \quad 1.50 \quad 40 \mathrm{C}$

$4.50 \quad$ I. $50 \quad 40 \mathrm{C}$

$4.50 \quad 1.50 \quad 40 \mathrm{C}$

4.50 I. 50 foc

$4.50 \quad I .50 \quad$ foC

$4.00 \quad$ I. $25 \quad 35 \mathrm{C}$

$6.50 \quad 2.00 \quad 65 \mathrm{C}$

$6.50 \quad 2.00 \quad 65 \mathrm{C}$

$6.50 \quad 2.00 \quad 65 \mathrm{C}$

$6.50 \quad 2.00 \quad 65 \mathrm{C}$

$6.50 \quad 2.00 \quad 65 \mathrm{C}$

$5.00 \quad 1.50 \quad$ foc

California Grown Seeds Mature Earliest and Bloom Most Luxuriantly. 


\section{CHAR L E C. N A V L E T C O M P A Y

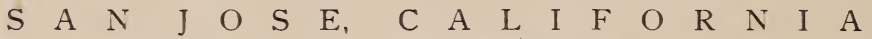 \\ NAVLET'S COSMOS \\ THE BEST IN THE WORLD

* PRICE LIST *

PRICES POST PAID

ib) th $1 / 4^{2} \quad \mathrm{OZ}$.

Early Dawn,.............. \$2.25 75C $25 \mathrm{C}$

New Early Blooming ........... $2.00 \quad 60 \mathrm{c} \quad 20 \mathrm{C}$

Mammoth, Perfection Pink......... 2.00 6oc $20 \mathrm{C}$

Mammoth, Perfection Red,........ 2.00 6oc $20 \mathrm{C}$

Mammoth, Perfection White,...... 2.00 6oc 20c

Mammoth, Perfection Mixed,....... 2.00 6oc $20 \mathrm{C}$

\section{NAVLET'S MIGNONETTE}

\section{THE BEST IN THE WORLD \\ PRICE LIST}

Allen's Defiance, for forcing $\ldots$ th $1 / 4$ th $\quad$ OZ.

Golden Macher, for cutting,....... I.75 5OC $20 \mathrm{C}$

Pure Machet, .............. I.75 $50 \mathrm{C} \quad 2 \mathrm{OC}$

Giant Pyramidal, ............... I.00 30 IOC

Common Sweet............... 40. I5 . $5 \mathrm{C}$

California Grown Seeds Mature Earliest and Bloom Most Luxuriantly. 


\section{CHARLES C. NAVLET COMPANY S A N J O S E, C A L I F O R N I A}

\section{NAVLET'S VERBENAS}

\section{THE BEST IN THE WORLD. * PRICE LIST *}

Mammoth Auricula-flowered. .........

Mammoth, Italian Striped.

PRICES POST PAID

Mammoth, Purple. .

it) I 4 th $\mathrm{Oz}$.

Mammoth, Pink.

$7 \cdot 50$

2.50

$75 \mathrm{C}$

$7 \cdot 50$

2.50

$75 \mathrm{C}$

7.50

2.50

$75 \mathrm{C}$

Mammoth,

$7 \cdot 50$

2.50

$75 \mathrm{C}$

Mammoth, Mixed.

8.00 $2: 50$

Soc

7.50

2.50

$65 \mathrm{C}$

California Grown Seeds Mature Earliest and Bloom Most Luxuriantly. 


\section{CHARLES C. NA VLET COM P A N Y $\begin{array}{lllllllllllllllll}S & A & N & J & O & S & E, & C & A & L & I & F & O & R & N & I & A\end{array}$}

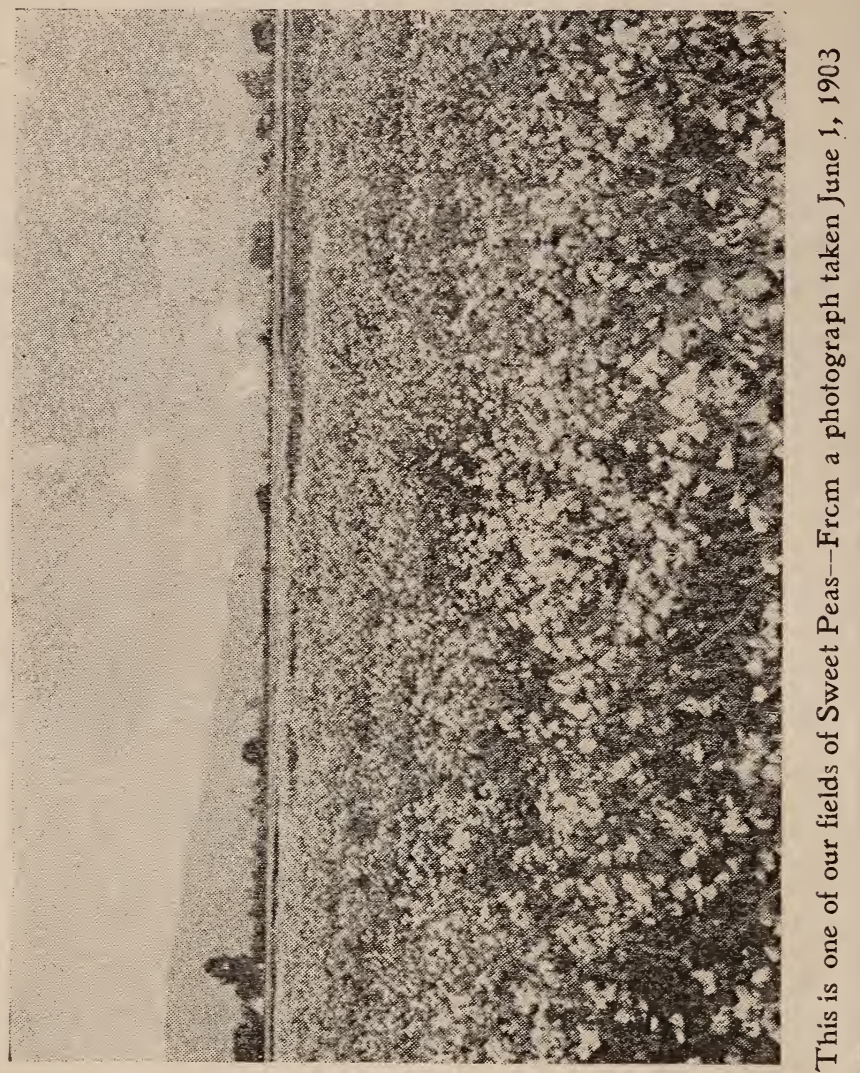

California Grown Seeds Mature Earliest and Bloom Most Luxuriantly. 


\section{IMPORTANT NOTICE}

While we exercise the greatest care to have all seeds pure and reliable, we do not give any warranty, expressed or implied. All contracts are subject to crop. F, O, B., San Jose.

CHARLES C. NAVLET COMPANY SEED GROWERS,

San Jose, - - - - - California 


\section{The prices listed in this Booklet}

will interest close figuring buyers -seedsmen who buy low or not at all. 